\title{
A high-altitude balloon experiment to probe stratospheric electric fields from low latitudes
}

\author{
Subramanian Gurubaran ${ }^{1}$, Manu Shanmugam ${ }^{2}$, Kaliappan Jawahar ${ }^{3}$, Kaliappan Emperumal ${ }^{3}$, \\ Prasanna Mahavarkar ${ }^{1}$, and Suneel Kumar Buduru ${ }^{4}$ \\ ${ }^{1}$ Indian Institute of Geomagnetism, Navi Mumbai 410 218, India \\ ${ }^{2}$ Tata Institute of Fundamental Research, Mumbai 400 005, India \\ ${ }^{3}$ Equatorial Geophysical Research Laboratory, Indian Institute of Geomagnetism, Tirunelveli 627 011, India \\ ${ }^{4}$ National Balloon Facility, Tata Institute of Fundamental Research, Hyderabad 500 062, India \\ Correspondence to: Subramanian Gurubaran (gurubara@iigs.iigm.res.in)
}

Received: 31 July 2016 - Revised: 11 January 2017 - Accepted: 12 January 2017 - Published: 3 February 2017

\begin{abstract}
The Earth's electrical environment hosts a giant electrical circuit, often referred to as the global electric circuit (GEC), linking the various sources of electrical generators located in the lower atmosphere, the ionosphere and the magnetosphere. The middle atmosphere (stratosphere and mesosphere) has been traditionally believed to be passively transmitting electric fields generated elsewhere. Some observations have reported anomalously large electric fields at these altitudes, and the scientific community has had to revisit the earlier hypothesis time and again. At stratospheric altitudes and especially at low latitudes, horizontal electric fields are believed to be of ionospheric origin. Though measurements of these fields from a balloon platform are challenging because of their small magnitudes (around a few $\mathrm{mV} \mathrm{m}^{-1}$ ), a suitably designed long-duration balloon experiment capable of detecting such small fields can provide useful information on the time evolution of ionospheric electric fields, which is otherwise possible only using radar or satellite in situ measurements. We present herein details of one such experiment, BEENS (Balloon Experiment on the Electrodynamics of Near Space), carried out from a low-latitude site in India. The instrument package for this experiment is comprised of four deployable booms for measurements of horizontal electric fields and one inclined boom for vertical electric field measurements, all equipped with conducting spheres at the tip. The experiment was conducted from Hyderabad $\left(17.5^{\circ} \mathrm{N}, 78.6^{\circ} \mathrm{E}\right)$ during the post-midnight hours on 14 December 2013. In spite of a few shortcomings we report herein, a noticeable feature of the observations has been the
\end{abstract}

detection of horizontal electric fields of $\sim 5 \mathrm{mV} \mathrm{m}^{-1}$ at the stratospheric altitudes of $\sim 35 \mathrm{~km}$.

Keywords. Ionosphere (electric fields and currents)

\section{Introduction}

The various sources of the electrical generators located in the lower atmosphere, the ionosphere and the magnetosphere and the currents flowing through them as well as between them and Earth constitute a giant electrical circuit often referred to as the global electric circuit (GEC; see Rycroft et al., 2012, for recent advances on this topic). The classical picture of the GEC incorporates the global thunderstorm activity as the only source, with the currents flowing from the thunderclouds charging the ionosphere to a few $100 \mathrm{kV}$ with respect to the Earth and the discharging currents leaking through the atmosphere over rest of the region. It was recognized some time ago that there are at least two other important sources that contribute to the global electric fields, namely the solar wind-magnetosphere dynamo that generates potential drop across the northern and southern polar ionospheric caps in the range of $30-150 \mathrm{kV}$ and the ionospheric dynamo originating in the $100-150 \mathrm{~km}$ altitude range due to tidal forcing that produces potential differences of 5 $10 \mathrm{kV}$ (Hays and Roble, 1979a, b).

In the past, the middle atmosphere was often considered to be electrodynamically passive, so that electric fields observed at stratospheric and mesospheric altitudes could be interpreted as those penetrating from above or from below. 
Solar-wind-induced magnetospheric convection, for example, produces large-scale fields of several tens of $\mathrm{mV} \mathrm{m}^{-1}$ in the polar cap and in the auroral ionosphere. These horizontal fields were indeed observed at balloon altitudes with little attenuation (see Mozer and Serlin, 1969, for example) in accordance with the results from a theoretical analysis (Park, 1976). In addition, large-scale ionospheric dynamo fields of a few $\mathrm{mV} \mathrm{m}^{-1}$ are expected to penetrate to stratospheric altitudes with little attenuation (Volland, 1972). It is to be noted that the global thunderstorm activity driving fair-weather electric fields is observed as vertical fields, whereas the upper atmospheric sources induce horizontal fields at balloon altitudes. In the case of the former, the Earth and the ionosphere act as equipotential surfaces, and the sources drive electric fields, which point essentially downward in the fairweather regions, i.e., far away from thunderstorm centers. In the case of the latter, the two upper atmospheric generators, namely the solar wind-magnetosphere-ionosphere dynamo and the ionospheric neutral wind dynamo, produce charge separation, resulting in large-scale electric fields, which are primarily in the horizontal direction. These horizontal fields map downward, as they are, to mesospheric and stratospheric altitudes.

Among the recent additions to our knowledge of the above processes, one spectacular phenomenon that deserves mention is the high-altitude discharge and the corresponding luminous events occurring above thunderstorms (Franz et al., 1990; Sentman et al., 1995; Rycroft and Odzimek, 2010, to state a few). Holzworth (1989) discovered the existence of large horizontal electric fields at stratospheric altitudes $(\sim 27 \mathrm{~km})$ from long-duration flights involving superpressure balloons. Until then, it was believed that the middle atmosphere essentially behaved as a passive element transmitting electric fields of lower and upper atmospheric sources. A later report by $\mathrm{Hu}$ and Holzworth (1997) confirmed the presence of these large electric fields over a wider latitude region of $30-75^{\circ}$ in the Southern Hemisphere. It was hypothesized then that atmospheric motions driven by an inertia gravity wave originating in the lower atmosphere could effectively induce horizontal electric fields in the range $10-20 \mathrm{mV} \mathrm{m}^{-1}$ during the night and $100-150 \mathrm{mV} \mathrm{m}^{-1}$ during the day, with the electric field vector undergoing a rotation at a rate equal to the period of the local inertia gravity wave. For inertia gravity waves with horizontal wavelengths of $\sim 500 \mathrm{~km}$, it follows that a corresponding inertial cell of size $500 \mathrm{~km}$ is capable of generating a $100 \mathrm{mV} \mathrm{m}^{-1}$ electric field, which would lead to a total potential drop of $\sim 50 \mathrm{kV}$ across the cell that would be comparable to the potential drop across the entire magnetosphere (Holzworth, 1995).

The first experiment of the Balloon Experiment on the Electrodynamics of Near Space (BEENS) carried out from the National Balloon Facility (NBF), Tata Institute of Fundamental Research (TIFR), Hyderabad $\left(17.5^{\circ} \mathrm{N}, 78.6^{\circ} \mathrm{E}\right)$, India, was of exploratory type with the primary objective of capturing electric field signals at stratospheric altitudes from low geomagnetic latitudes $\left(\sim 8-9^{\circ}\right)$. It is generally believed that fields other than those driven by thunderstorm sources in the lower atmosphere are weak $\left(1-3 \mathrm{mV} \mathrm{m}^{-1}\right)$ and are of ionospheric origin at such low geomagnetic latitudes. Advances in femtoampere bias current field-effect transistor amplifier technology and some simple circuit ideas that emerged to address the problem of minimizing the leakage currents had motivated us to carry out measurements of such weak electric fields. The experiment was conducted during the early hours of 14 December 2013, using a $110000 \mathrm{~m}^{3}$ balloon that reached a float altitude of $35 \mathrm{~km}$.

In Sect. 2, a brief description of the BEENS experiment and the instrumentation involved is presented. Sketches showing the gondola, as well as the central body, and the alignment of probes as per their configuration chosen for this experiment are provided. Payload electronics are discussed in this section with the help of a schematic showing the major circuit elements and the signal flow. As shown in this section, a thermal infrared image available from the EUMETCast data archival center revealed fair-weather conditions across the Indian Peninsula at the time of launch. The first results from this experiment are presented in Sect. 3. Reliable measurements of vertical electric field were not possible due to the five-probe configuration and the kind of deployable boom incorporated for the vertical probe, thereby leaving us with a meaningful interpretation to be made with the measurements from the horizontal probes alone. During the flight, we also encountered change in the DC offset in the electric field measurements and enhancement in signal amplitudes possibly caused by the DC torque motor used for spinning the payload. These aspects are discussed in this section. Finally, in Sect. 4 we present a discussion on the possible sources of the observed electric fields.

\section{Description of the BEENS experiment}

Employing the double-probe technique, which is well discussed in the literature (e.g., Holzworth and Bering III, 1998, and references therein), the BEENS payload mounted on a gondola comprised two orthogonal pairs of spherical probes, namely $\mathrm{P}_{1}-\mathrm{P}_{2}$ (referred to as $x$ probes) and $\mathrm{P}_{3}-\mathrm{P}_{4}$ (referred to as $y$ probes), with the line joining the probes $\mathrm{P}_{1}$ and $\mathrm{P}_{2}$ defining the payload $x$ axis and the line joining the probes $\mathrm{P}_{3}$ and $\mathrm{P}_{4}$ defining the payload $y$ axis (see Fig. 1a for the geometry of the probe alignment). The reference magnetometer was mounted on the top deck of the central body in such a manner that its two horizontal sensors were aligned along the two horizontal payload axes defined above. The probes deployed in the horizontal plane were configured to yield measures of the north-south and east-west components of the horizontal electric field. The fifth probe $\left(\mathrm{P}_{5}\right)$ was mounted at the end of another boom that was deployed at an angle of $45^{\circ}$ to the horizontal plane. In this manner, this probe, along with one of the probes deployed in the payload $x$ axis, namely $\mathrm{P}_{1}$, 


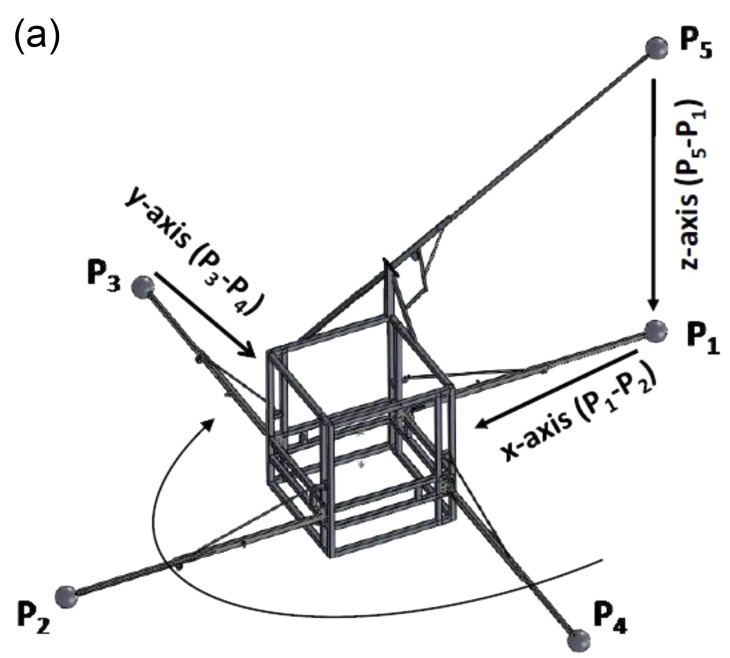

(b)

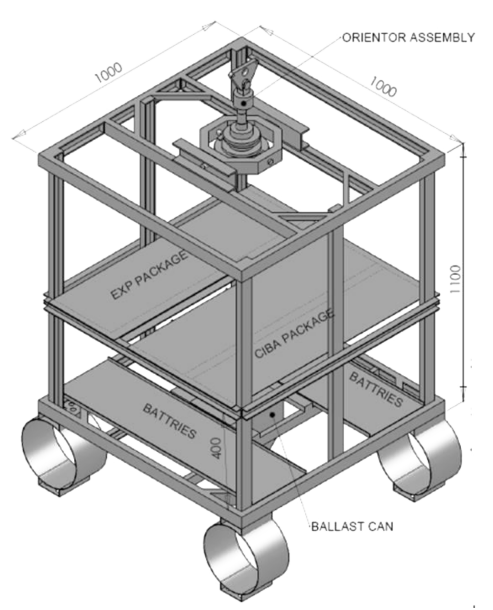

Figure 1. (a) A sketch of gondola (central body or the payload) and the booms shown in deployed condition (not to scale). The directions of the three axes referenced with respect to the probe configuration are indicated. The curved line with an arrow at its tip indicates the direction of the rotation of gondola. The dimensions of the gondola are given in (b). Details regarding the individual lengths of the booms are provided in the text. (b) A drawing of the gondola showing the locations of various subsystems (courtesy of the National Balloon Facility, TIFR, Hyderabad).

was configured to yield a measure of the vertical electric field $\left(\boldsymbol{E}_{z}\right)$ (the pair $\mathrm{P}_{5}-\mathrm{P}_{1}$ will hereafter be referred to as $z$ probes).

All five probes were identical $20 \mathrm{~cm}$ diameter aluminum spheres coated with Aquadag, a conducting carbon paint used to minimize the work function differences between them. Each of the four booms to where the horizontal $x$ and $y$ probes were mounted was $3 \mathrm{~m}$ long, with its outermost $1 \mathrm{~m}$ made of insulating fiberglass rods and was kept in stowed condition prior to the launch. With the gondola dimensions of about $1 \mathrm{~m}$ each on all sides (see Fig. $1 \mathrm{~b}$ for a drawing of the gondola showing the locations of the various subsys-

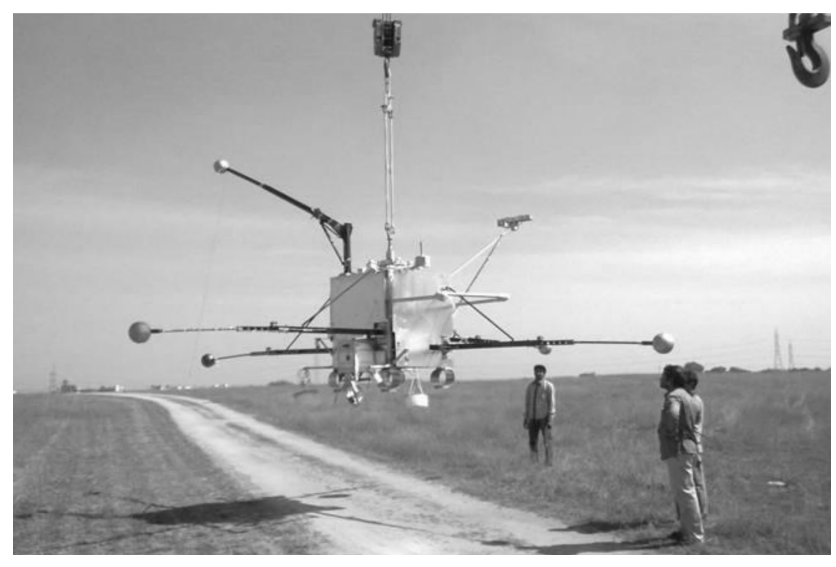

Figure 2. A photograph showing the payload undergoing field tests. The payload rotation was carried out for several hours and the stability of the platform was ensured during these tests.

tems), the probe-to-probe baselines achieved for the $x$ and $y$ probes were 6.92 and $7.02 \mathrm{~m}$, respectively. The probe-toprobe baseline achieved in the vertical direction was $2.82 \mathrm{~m}$.

A DC torque motor, referred to hereafter as orientor, enabled the rotation (clockwise) of the gondola about the vertical axis for meaningful horizontal electric field measurements. A universal coupler immediately following the orientor provided additional stability for the platform by ensuring that the axis of rotation did not get tilted as the balloon maneuvred through and around the ambient medium during its flight. Ground tests prior to launch yielded a rotation rate in the range of $1-2 \mathrm{rpm}$. The orientor did not have a control arrangement for this experiment, and so it was anticipated that the rotation rate would increase as the balloon ascended to higher altitudes (due to lower friction with the ambient gas higher up), which indeed was the case. Figure 2 depicts a photograph of the payload undergoing rigorous field tests with the booms having been kept in the deployed condition.

The three-axis fluxgate magnetometer (FGM) serving as a reference magnetometer or magnetic compass for the electric field measurements as mentioned earlier and a two-axis search coil magnetometer (SCM) for monitoring fluctuating magnetic fields in the ELF-VLF frequency range completed the instrument package. The search coil sensors were mounted at the end of a rigid $1.5 \mathrm{~m}$ long boom. The four sides (of $\sim 1 \mathrm{~m}^{2}$ area) of the gondola were mostly covered by aluminum panels with Teflon spacers, which electrically isolated the panels from the gondola. These aluminum panels were also coated with Aquadag. With their large conductive area $\left(\sim 3.6 \mathrm{~m}^{2}\right)$, the panels provided a common ground. Considering that the covered area was 2 orders of magnitude greater than the probe surface area $\left(\sim 0.0314 \mathrm{~m}^{2}\right)$, it was felt that this would be sufficient enough for a reliable measurement of electric field.

Figure 3 depicts a block diagram of the experimental setup of the BEENS payload. A specially designed three-channel 


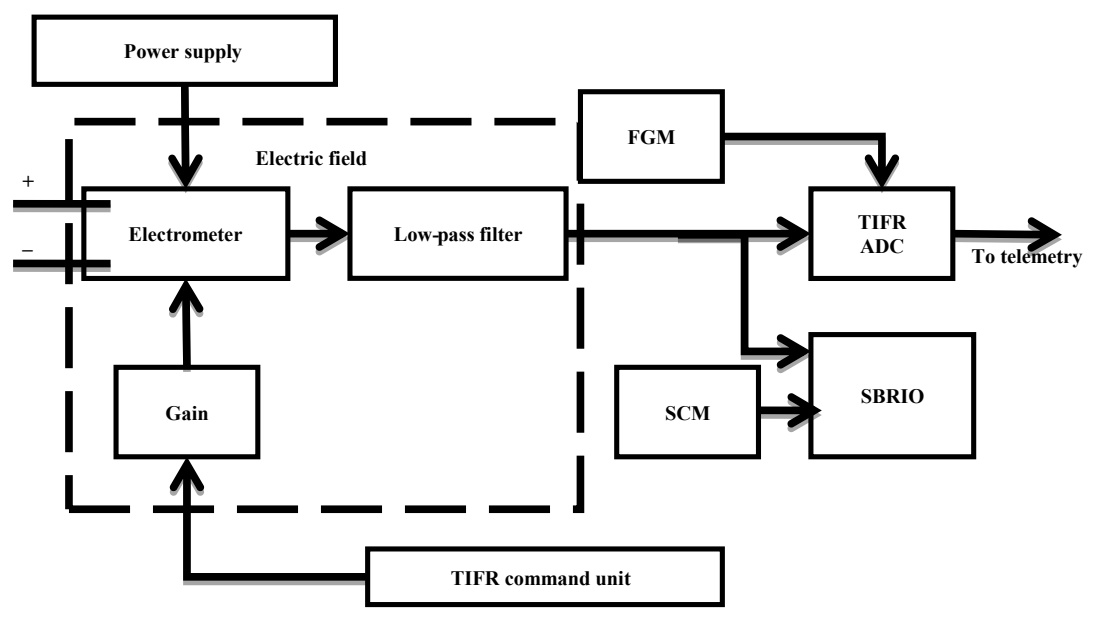

Figure 3. A block diagram of the experimental setup of the BEENS payload.

differential electrometer with ultra-high-input impedance permitting ultra-low-input bias current $\left(\sim 3 \mathrm{fA}\right.$ at $\left.25^{\circ} \mathrm{C}\right)$ was used to sense the difference voltages between the probe pairs. Shielded cables brought the high-impedance input signal directly to the inputs pins of an INA116 instrumentation amplifier operated in a differential mode. Guard voltages were inherently generated within the instrumentation amplifier and were available for driving the outer conductors of the shielded cables to the floating potential of the corresponding probe. This arrangement combined with the way the wires were soldered directly in air on to the input pins of INA116 amplifier enabled considerable reduction of any stray parasitic capacitance along the signal path. Further, with a single external resistor, a corresponding gain setting could be achieved with this instrumentation amplifier. Any circuit complications were greatly minimized with the optimum use of a single instrumentation amplifier for each of the electric field channels, which would have the advantage of carrying out all three functions mentioned above, i.e., differencing, generating the guard voltage and providing a gain setting (see Jawahar et al., 2016, for further details of the electrometer circuit).

One of the design goals of the above circuit was to capture high-frequency (a few $\mathrm{Hz}$ to few tens of $\mathrm{kHz}$ in the ELF and lower VLF range) signals, in addition to the slowly varying DC electric field. To meet this requirement, provision was made for a second-order low-pass filter with cutoff frequency that could be chosen to be 1 or $32 \mathrm{kHz}$ depending on the frequency range of interest. Further, the single-board version of an embedded control and acquisition unit (referred to as a single-board reconfigurable input/output (SBRIO) device) of National Instruments was included as part of the instrument package to record high-frequency data (refer to Fig. 3). The SBRIO could monitor and record high frequency electric field signals with its sampling rate of $200 \mathrm{kS} \mathrm{s}^{-1}$ and onboard storage option. The electrometer output was simultaneously routed to the 12 bit ADC input of an onboard telemetry encoder. In this work, we examine only the telemetered doubleprobe data collected at slow sampling rates, which would essentially represent the DC electric field at the probing balloon altitudes.

Within the flight encoder, the output from the three electric field and the three magnetic field channels was converted to a single serial digital data stream for transmission to the ground station. With a differential input in the range of $+4.7 \mathrm{~V}$, the 12 bit ADC resolution would enable the measurement system to record a change of $\sim 2.3 \mathrm{mV}$ minimum (equivalent to a horizontal electric field of $\sim 0.3 \mathrm{mV} \mathrm{m}^{-1}$ ). The raw data were sampled at $\sim 2.25 \mathrm{~ms}$ time interval. The encoder was programmed to transmit 32-channel serial data, which, therefore, resulted in every channel being sampled at $\mathrm{a} \sim 72 \mathrm{~ms}$ time interval (or $13.8 \mathrm{~Hz}$ sampling frequency). As mentioned earlier, the rotation of the payload would induce a sinusoidal signal of the electric field at a period equal to the payload rotation period. On this count, the sampling frequency of $13.8 \mathrm{~Hz}$ for the electric field channels noted above would be sufficient given that the smallest gondola rotation rate was $0.16 \mathrm{~Hz}$ (corresponding to a period of $6 \mathrm{~s}$ ), as we will see later.

Ground verification of the payload electronics was carried out on the ground in advance, wherein each pair of probes was tested by applying DC potentials (one of the probes was given positive voltage and the other grounded) and the output monitored on the corresponding telemetry channel. The results from one such test performed for the $x$ and $y$ probes just prior to launch are presented in Fig. 4. The linear response of the electrometer output for various probe voltages is clearly evident in Fig. 4, indicating the anticipated performance of the electric field channels prior to launch. We need to mention here that, apart from ground tests and verification, there was no provision made in the present experiment for onboard calibration of the probe-electrometer system. 

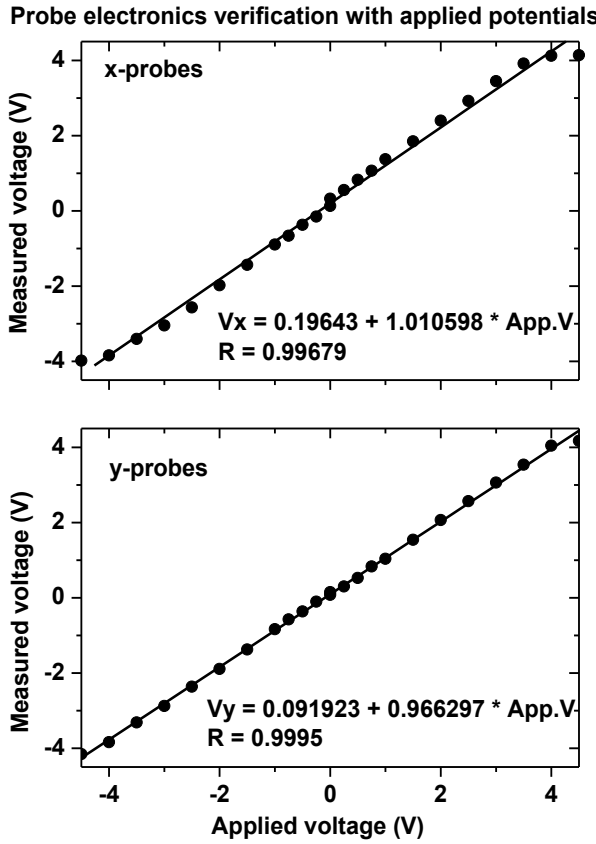

Figure 4. Performance results for the probe-electrometer system obtained just prior to launch. Known voltages are applied to the $x$ and $y$ probes separately in isolation and the electrometer outputs monitored on the respective telemetry channels. The equation for the linear regression line and the correlation coefficient $(R)$ are mentioned separately in each plot.

A load line of about $75 \mathrm{~m}$ was used to connect the balloon with the payload. The main packages along the load line were a cut-off device situated just below the balloon, a parachute, a GPS radiosonde and a radar reflector. The support packages for the BEENS experiment were the telemetry unit, a telecommand unit, a ballast container, a battery pack and other housekeeping devices. The launch was cleared after critical assessments of the functioning of all subsystems, including the payload, were carried out through continuous long-duration ground tests.

It was expected that the effects of any electrical noise created by the balloon movement through the ambient atmosphere would be overcome by the long load line and the electrical isolation of the probes. Further, any balloon-induced noise would appear as common-mode noise, which would be eliminated because of the differential measurements.

Several flights have been conducted in the past from the same location during the Indian Middle Atmosphere Program in the 1980s (Gupta and Narayan, 1987; Garg et al., 1989; Sampath et al., 1989; Udare et al., 1991). Most of those flights were aimed at making measurements of polar conductivities, ion densities and mobilities, though a few of them yielded vertical electric field profiles during the balloon ascents.

The balloon launch with the BEENS payload took place at 00:50 Indian Standard Time (IST), which is $5.5 \mathrm{~h}$ ahead

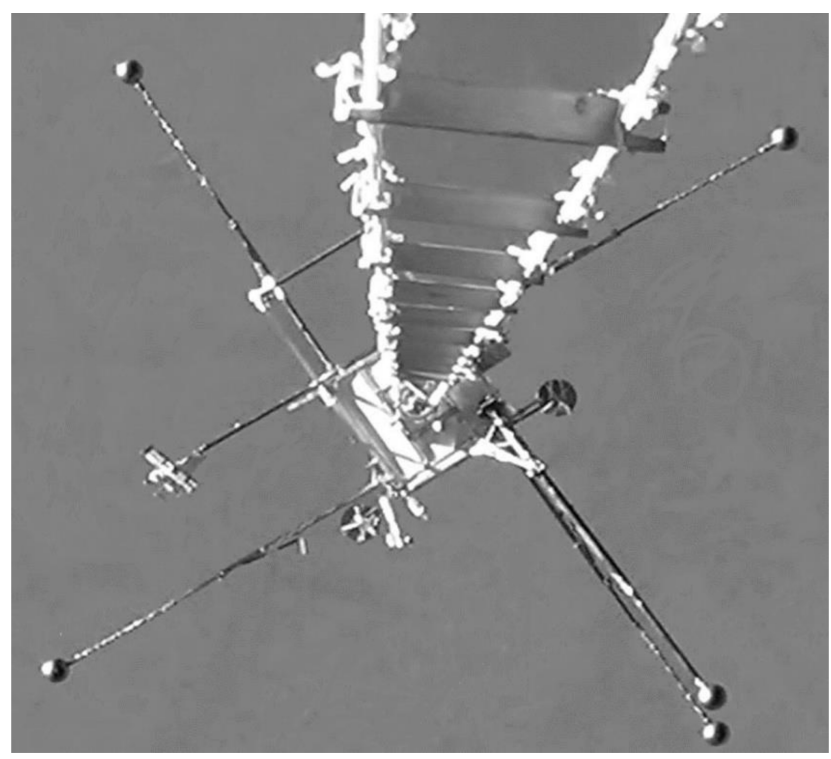

Figure 5. A snapshot of the payload viewed from the load line hanging from the balloon.

of Greenwich Mean Time, on 14 December 2013, after being postponed twice on two subsequent days due to unfavorable surface wind conditions. Immediately after the release of the balloon, a boom deployment command was given and all five booms were successfully deployed one after another. Another command given $16 \mathrm{~min}$ after the launch activated the orientor and the payload started rotating as evidenced in the reference magnetometer data telemetered to the ground. In Fig. 5, we present a snapshot of the payload during a late stage (early morning hours) of the flight with booms seen in the deployed state.

The orography, specific to the track along which the balloon drifted, is contributed primarily by the Deccan Plateau, a region elevated in the range of $300-500 \mathrm{~m}$ above mean sea level. There are no mountain ranges with altitudes greater than $1000 \mathrm{~m}$ in the vicinity of at least $300 \mathrm{~km}$ from the balloon track on all sides. The EUMETCast data archival center provided access to the image archives from the EUMETSAT fleet of satellites. A thermal infrared (10.2-12.5 $\mu \mathrm{m})$ image of the Indian subcontinent obtained from one such archive is depicted in Fig. 6. This image, which was captured at 02:30 IST on 14 December 2013, does not reveal any highaltitude convective cloud system in the vicinity of the balloon path (shown as a thick red curve).

When the balloon reached the float altitude of $\sim 35 \mathrm{~km}$, the stratospheric winds in that region were blowing with speeds of $\sim 20 \mathrm{~m} \mathrm{~s}^{-1}$ towards west. With the safe flight corridor for the recovery of the payload being about $400 \mathrm{~km}$ on the west, the flight had to be terminated at around 08:00 IST after achieving a float duration of slightly more than $4 \mathrm{~h}$. Figure 7 depicts the GPS tracked balloon altitude (bottom panel) and the wind speed and wind direction measured by the GPS 


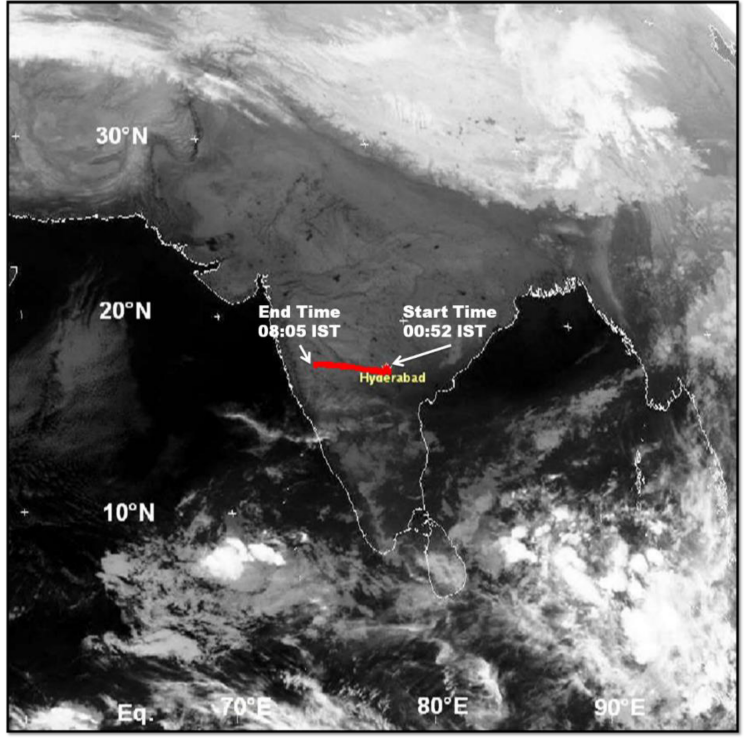

Figure 6. A thermal infrared image (10.5-12.5 mm) obtained from the EUMETSAT's fleet of satellites at 02:30 IST on 14 December 2013. The balloon track is indicated in red.

radiosonde (top panel). As noticed herein, the wind direction remained at $\sim 90^{\circ}$ (which is easterly) between 03:30 and 08:00 IST, when the balloon was at the float altitude. The westward drift of the balloon continuously over time (shown as red curve in Fig. 6) was essentially due to the easterly winds prevailing during this period at high altitudes.

As mentioned earlier, there was a provision kept to change the gain of the electrometer for any of the three channels by the choice of a suitable resistor through a telecommand. As soon as the balloon approached the float altitude, a command was given from the ground for the $x$ and $y$ electrometer voltage gain to increase by 10 times. The change was immediately noticed in the telemetry channels. Until then, little variation in the signal level was seen on the computer monitor in real time. Immediately after the implementation of a 10fold gain, the two probe outputs started displaying variations that appeared to be in tune with the rotation of the payload. Further, as the balloon approached its float altitude, clear sinusoid-like variations could be seen on the real-time display unit, indicating the presence of an ambient stratospheric electric field.

Deduction of the horizontal electric field at balloon altitudes first involves careful examination of raw data for every payload rotation. The magnetometer sensors were configured in such a manner that the $x$ sensor output led the $y$ sensor output when the payload rotated in a clockwise direction. It should be noted here that the labels $x$ and $y$ were nothing to do with the direction (north-south/east-west) and were arbitrarily assigned to the pairs of probes for the given geometry. Figure 8 depicts a 40 s sample of the magnetic data (top panel) revealing that the $x$ channel led the $y$ channel by $90^{\circ}$

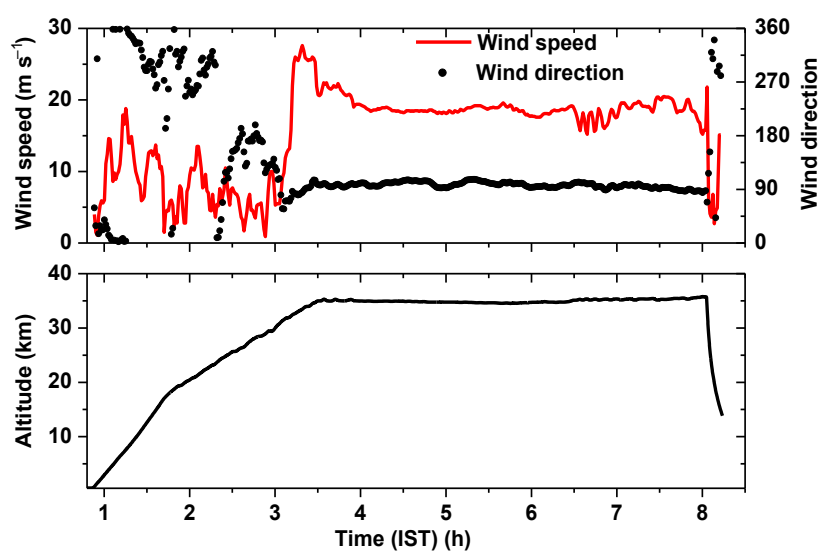

Figure 7. The location of the BEENS payload, when plotted altitude versus time, during the experiment (bottom panel) and the corresponding ambient wind speed and wind direction recorded then (top panel).

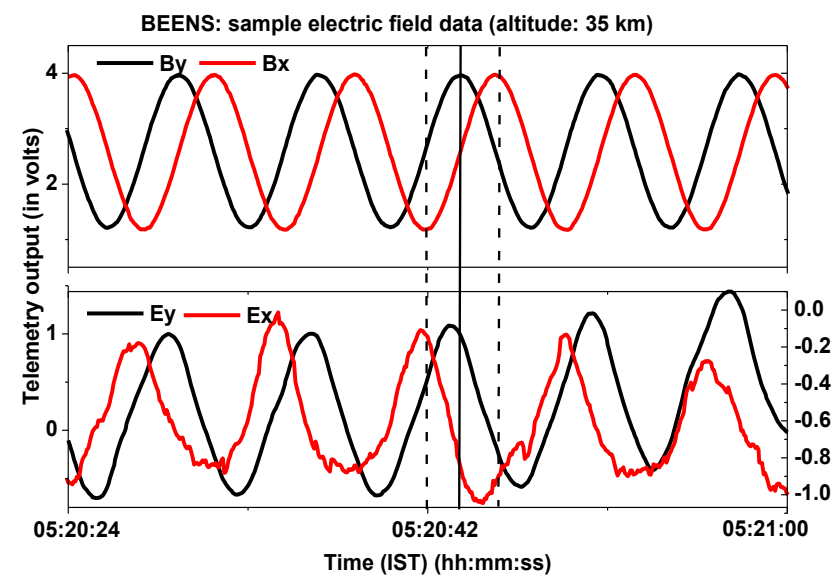

Figure 8. The raw magnetic field data, $\boldsymbol{B}_{x}$ and $\boldsymbol{B}_{y}$ from the $x$ and $y$ sensors, respectively (top panel), and the corresponding electric field channels.

(one-fourth of the payload rotation period). In terms of magnetic field units, the output range of 1.148-3.998 V in Fig. 8 (top panel) was referenced to $2.5 \mathrm{~V}$ and hence would correspond to a range of -1.352 to $1.498 \mathrm{~V}$. As the sensitivity of the magnetometers was $36.5 \mu \mathrm{VnT}^{-1}$, a full range of $2.85 \mathrm{~V}$ peak to peak corresponds to a magnetic field in the neighborhood of $78082 \mathrm{nT}$ peak to peak or an amplitude equivalent to $39041 \mathrm{nT}$, which is roughly the value of the Earth's magnetic field at this location.

With regard to the electric field measurement, as mentioned in Sect. 2, the $y$ probes were aligned parallel to the $y$ sensor of the magnetometer, whereas the $x$ probes were aligned anti-parallel to the $x$ magnetometer sensor. Due to this arrangement, the measurement of the $x$ probes would have to be shifted in phase by $180^{\circ}$. In any case, the payload rotation ensured that the ambient electric field appeared as a sinusoidal signal as can be seen in the telemetry data for 
the electric field probes shown in Fig. 8 (bottom panel). The continuous vertical line indicates the near alignment of the $y$ channels of electric and magnetic field probes, whereas the dashed line reveals the near $180^{\circ}$ phase difference between the respective $x$ channels mentioned above. For the data from the electric field channels plotted in Fig. 8, we had used 25point running means of the raw telemetry outputs. Further description of the data will be presented in the next section.

We use the traditional harmonic analysis and the local version of the harmonic analysis, namely the complex demodulation technique, for the estimation of horizontal electric field from the BEENS telemetry data. For every payload rotation, the time period is determined from the magnetometer data and the harmonic analysis is applied to the electric field data to estimate the amplitude and phase of the horizontal electric field in a least-squares sense. The phase, computed separately for the $x$ and $y$ probe outputs, would represent the direction of the electric field vector. The magnetometer $x$ channel would serve as a reference for the estimation of phase from $x$ probe outputs, whereas the magnetometer $y$ channel would serve as a reference for the phases estimated from $y$ probe outputs.

The complex demodulation technique is more flexible when it comes to data for which the payload rotation rate or the amplitude or the phase varied and when the data cannot be expressed simply in terms of cosine functions. The complex demodulation has been recognized to be the local version of the harmonic analysis (Bloomfield, 2000). The analysis adopted for the present analysis invokes demodulation at a chosen center frequency followed by low-pass filtering. The method is well discussed in Bloomfield (2000) and will not be repeated here.

\section{Results}

In this section we examine the behavior of the three components of the stratospheric electric fields. As mentioned earlier, the spinning of the payload about its vertical axis enabled useful determination of the horizontal components of the electric field, as any differences in contact potentials between the probes and DC offsets, if any, would appear as quasi-DC shifts in the probe outputs. The local horizontal electric field vector would then appear as a quasi-sinusoidal variation in each of the two orthogonal probe outputs with a period exactly equal to the period of rotation of the gondola about its axis.

The temporal variation in the two orthogonal components (recorded as sinusoids) of the ambient magnetic field recovered from the reference magnetometer data had a sinusoidal nature, and this was used to compute the payload rotation rate. As can be noted in Fig. 9, the payload rotation stabilized only after the balloon attained the float altitude of $\sim 35 \mathrm{~km}$ at $\sim$ 03:30 IST. The period of rotation reached the lowest value of $\sim 6 \mathrm{~s}$ (corresponding to $\sim 10 \mathrm{rpm}$ ), increasing to around

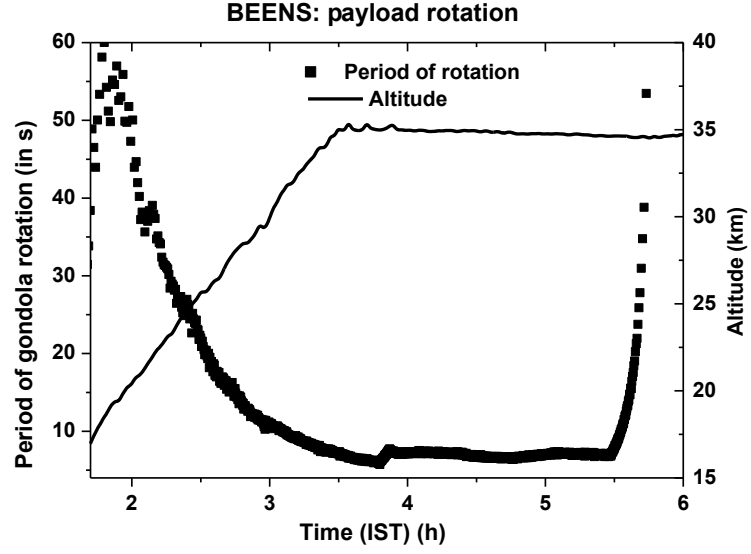

Figure 9. The payload rotation rate (squared symbols) computed using the reference magnetometer data and plotted along with the balloon altitude (continuous curve).

$\sim 60 \mathrm{~s}$ at 02:00 IST. Unfortunately, the orientor responsible for the payload rotation malfunctioned at 05:30 IST, following which the rotation was disabled. For these reasons, we considered only about $90 \mathrm{~min}$ of data, i.e., between 04:00 and 05:30IST, for estimating the horizontal component of the electric field.

Before we present our results, a few points regarding the impact of the five-probe configuration and the inclined boom on the veracity of the vertical electric field measurements will be in order. The boom that was used to deploy $\mathrm{P}_{5}$ (refer to Fig. 1a) did not have an insulating component at its outer end like the ones used for the other four probes, though the entire boom was electrically isolated from the gondola and the ground panels with Teflon spacers. The potential differences measured in the vertical direction were later found to be greatly influenced by this arrangement. Figure 10 depicts the $z$ probe difference output for times when the balloon ascended. We should note here that the $z$ pair of probes could not be calibrated on ground with known potentials as was done for the horizontal pair of probes due to the large ambient atmospheric vertical electric field present. At this stage, we do not intend to take the discussion about the vertical electric field measurements any further for the simple reason that they are not reliable.

Another issue pertaining to the measurements was to do with the requirement of the probe geometry, wherein one of the $x$ probes was to be used for the vertical electric field measurement. In fact, out of the two $x$ probes $\left(\mathrm{P}_{1}\right.$ and $\left.\mathrm{P}_{2}\right)$, probe $\mathrm{P}_{1}$ was reckoned to measure a - Ve potential for the $\mathrm{P}_{5}-\mathrm{P}_{1}$ pair and therefore connected to the negative input of the $z$ instrumentation amplifier and was reckoned to measure $\mathrm{a}+\mathrm{Ve}$ potential for the $\mathrm{P}_{1}-\mathrm{P}_{2}$ pair and connected to the positive input of the $x$ instrumentation amplifier. As can be appreciated, there was an interconnection that was to be realized between the inputs of $x$ and $z$ channels. With this arrangement, any gondola noise present in the $z$ channel was likely to couple 


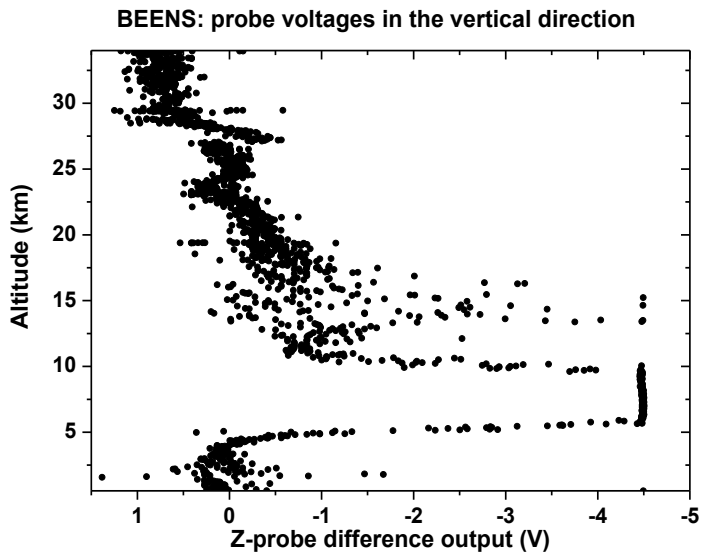

Figure 10. Difference output from the $z$ probes $\left(\mathrm{P}_{5}-\mathrm{P}_{1}\right.$ pair).

to the $x$ channel. In Fig. 11, we show the nature and extent of coupling of these two channels for a data set spanning $2 \mathrm{~min}$ between 03:46 and 03:48 IST. No discernible electric field signature varying at the gondola rotation rate can be inferred from the $x$ channel at these times. Rather, the $x$ channel is seen to track the $z$ channel output. The inadvertent manner in which the electrical interconnections were realized for this five-probe configuration resulted in the cross-coupling between the $x$ and $z$ channels, implying that the derived amplitudes of the ambient electric field would be underestimated to a large extent. However, about $30 \mathrm{~min}$ later, when the balloon reached a stable float altitude (refer to Fig. 9), the $x$ channel showed sinusoidal-like variations but with reduced amplitudes for reasons not known yet. In contrast, the $y$ probes were isolated electrically and distanced well away from the booms used to deploy other probes (refer to Figs. 1a and 5). Because one pair of probes is sufficient for estimating horizontal electric fields on a rotating platform, we consider only the $y$ channel telemetry output for that purpose in the rest of the paper.

Before we examine the data sets, we caution the readers about a possible electrical/electromagnetic noise caused by the DC torque motor and the power supply driving the motor, which could have impacted the measurements. At 03:48 IST, the power supply to the orientor was switched off by a ground command (at 03:48 IST) for a brief interval of $\sim 3$ min to assess the performance of the electric field channels then. The magnetometer output revealed that the payload continued to spin at the same rate due to its inertia, though the supply to the motor was switched off. During this time interval, the electric field channels registered a considerable change in the DC offset and we noticed nearly a 2 -fold decrease in the signal amplitude in the $y$ channel.

In Fig. 12, we show the $y$ channel output, wherein we depict the 25-point running means plotted in electric field units of $\mathrm{mV} \mathrm{m}^{-1}$, along with the demodulated signal representing the electric field. The electric field values were obtained af-

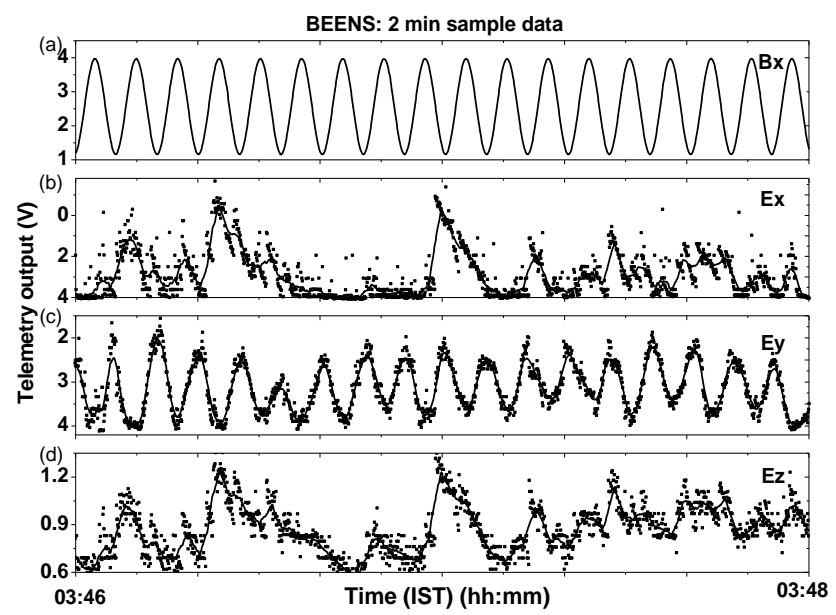

Figure 11. The raw outputs from the $x, y$ and $z$ probe pairs (b-d) along with the magnetometer $x$ channel output (a).

ter dividing the difference output voltage by the physical distance between the corresponding probes. It can be readily seen that the DC offset of this channel decreased from $\sim 45$ to $\sim 5 \mathrm{mV} \mathrm{m}^{-1}$. Further, the amplitude of the electric field signal was reduced from $\sim 8$ to $\sim 5 \mathrm{mV} \mathrm{m}^{-1}$ during this period, as can be seen in the bottom panel of Fig. 12. As soon as the power supply to the DC torque motor was switched on, both DC offset and the signal amplitude reached their earlier values (not shown here). We do not know yet how the motor or its power supply could have caused the subtle variations noticed in the data. On the other hand, it makes sense to treat the difference output at times when the motor was switched off as free from the motor-induced noise and use them to scale down the electric field values just outside this time interval. Further, the scaling factor of $\sim 2.4$ so obtained was used for the remaining data set for the entire 90 min duration. In this process it is assumed that the phases did not vary, though the amplitudes were scaled down. Sample electric field data at the float altitude of $\sim 35 \mathrm{~km}$ for three different 2 min time segments, namely 03:37-03:39, 04:3704:39 and 05:25-05:27 IST, are depicted in Fig. 13. The data points have been smoothed with 25 -point running means that correspond to windows of $1.8 \mathrm{~s}$ time duration. The electric field modulated by the payload rotation can be clearly seen in these data sets. Before proceeding to estimate the electric fields, the raw data for the above duration were filtered with a pass band in the range of 5.6-7.3 s, corresponding to the range over which the payload rotation period varied. With this process, the DC offsets and any of their slowly varying components were removed in advance.

We now elaborate the procedure adopted for meaningful interpretation of the amplitude and phase of the electric field vector estimated for every payload rotation or cycle. The first step involved the use of the magnetometer $y$ channel data to compute the period of the payload rotation for a particular 


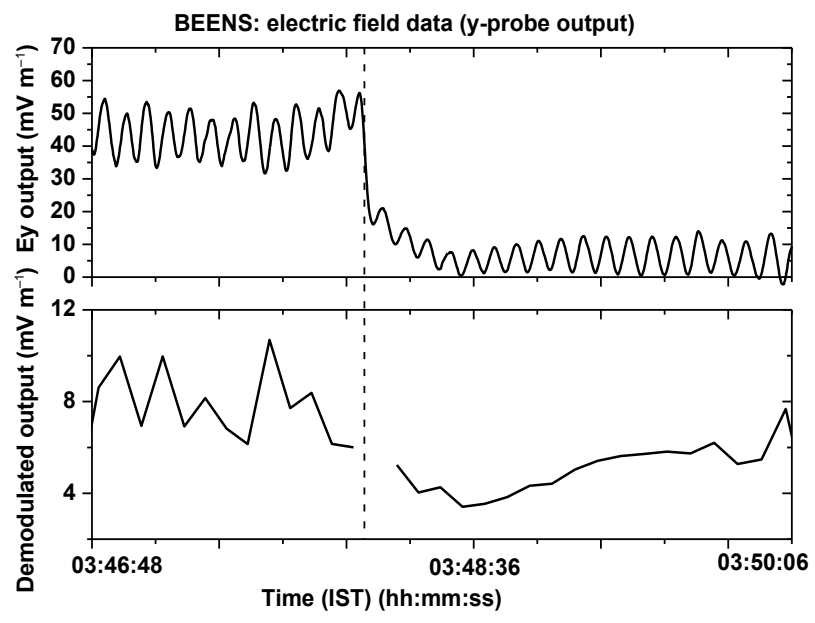

Figure 12. The $\boldsymbol{E}_{y}$ channel output (top panel) (converted to electric field units) for a brief interval of time when the DC torque motor was switched off. In the bottom panel, the demodulated signal is shown. The dashed vertical line represents the time (03:48 IST) when the DC torque motor was turned off. For further details, see the text.

instant. The same data set also served to denote the reference time for the electric field channels for the corresponding cycle. In specific terms, the positive peak of the magnetometer $y$ channel data would define the beginning of the time or "time zero" or, equivalently, the position of the gondola with its angular measure equal to $0^{\circ}$ for that particular cycle and the subsequent times for the respective cycle would be reckoned with reference to that instant. The following magnetometer $y$ channel peak would define the "time zero" for the next cycle and so on. The electric field data for every cycle, with time so defined, were separately analyzed using the harmonic analysis, and the amplitudes and phases were estimated in the least-squares sense. As per the convention adopted, the phase of the electric field vector would refer to the time when the electric field reaches its maximum value within a particular cycle. While depicting the results, we convert the time to angular measure (clockwise from geomagnetic north), reckoning one payload cycle to cover $360^{\circ}$. With geomagnetic north as a reference, the phase of the electric field vector thus defines the position of the electric field vector in space. We repeat the above procedure separately for the $x$ probe outputs but with magnetometer $x$ channel serving as a reference.

In Fig. 14, the amplitude (panel a) and phases (panel b) of the electric field estimated from the band-pass-filtered data for the $y$ probe outputs over the selected time interval are depicted. As mentioned earlier, this 90 min duration was chosen considering the fact that the payload rotation during this period was stable and the rotation rate was nearly constant. There were $\sim 600$ payload rotations during this period, with each rotation leading to a measurement of electric field vector in the horizontal plane of the payload. The amplitude of

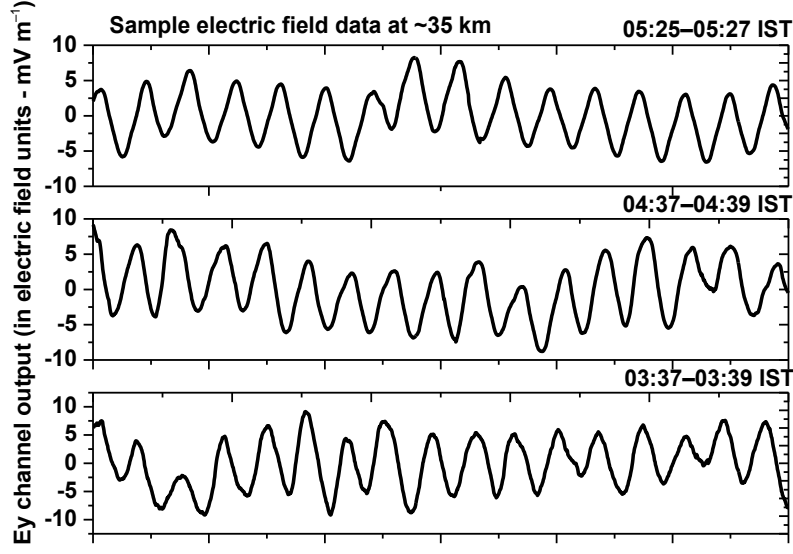

Figure 13. Sample electric field data at the float altitude of $35 \mathrm{~km}$ for the three different 2 min time segments. The DC offsets were removed in this plot.

the electric field estimated from the $y$ probes was in the range of 4-6 $\mathrm{mV} \mathrm{m}^{-1}$ during the period considered for analysis.

Though the amplitudes derived from the $x$ probe outputs (not shown here) were underestimated due to the distortion induced by the $z$ probe, the phases for the $x$ channel were stable over the last $1 \mathrm{~h}$ observation duration, as can be seen in the bottom panel of Fig. 14. It may be noted that both $x$ and $y$ channel phases were referenced with respect to geomagnetic north and therefore indicate the direction that the electric field vector would point at any instant of time. The observation that both $x$ and $y$ probes measured nearly the same phase confirms that the $x$ and $y$ probes were indeed sampling the same ambient electric field.

An interesting inference to be made from the bottom panel depicted in Fig. 14 is that the electric field was predominantly in the zonal direction and directed towards west before 04:30 IST, when their "phases" were around $270^{\circ}$. The electric field turned clockwise later between 04:30 and 05:00 IST, and was directed northwestward with their "phases" showing values around $330^{\circ}$. When the $x$ probe outputs were more stable during the last $1 \mathrm{~h}$ of useful electric field observation, one can notice similar behavior, and it was therefore consistent with the direction of the electric field inferred from the $y$ probe output.

Finally, we show the geomagnetic north-south $\left(\boldsymbol{E}_{\mathrm{NS}}\right)$ and geomagnetic east-west $\left(\boldsymbol{E}_{\mathrm{EW}}\right)$ components of the electric field vector computed from the $y$ probe outputs for the $90 \mathrm{~min}$ duration in Fig. 15. These were the 5 min averages of the estimated electric field components with the error bars representing their standard deviation within the respective 5 min block. The electric field was largely westward until 04:30 IST but later changed direction to northwestward. In Fig. 15 we notice $\sim 5 \mathrm{mV} \mathrm{m}^{-1}$ northward field and somewhat weaker $\sim 3 \mathrm{mV} \mathrm{m}^{-1}$ eastward field, with the standard deviations in the range of $0.5-1.0 \mathrm{mV} \mathrm{m}^{-1}$. 


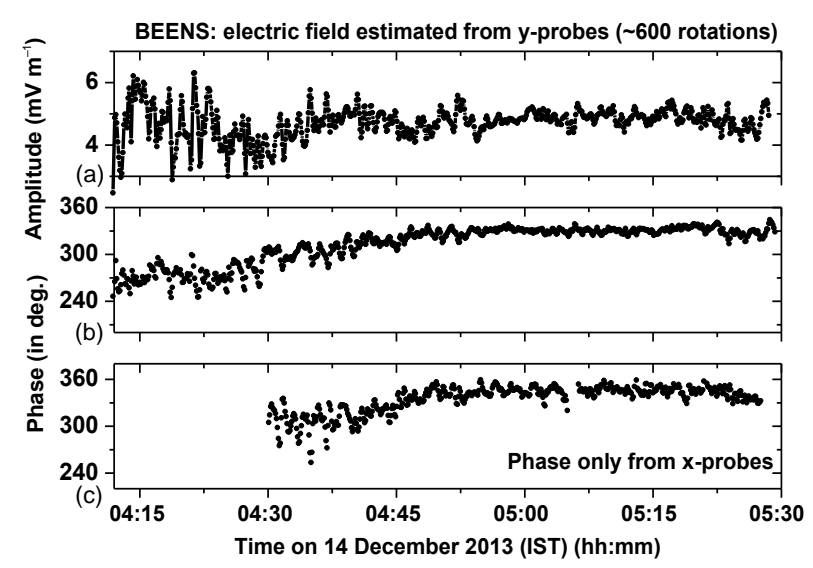

Figure 14. The magnitude and direction (a, b, respectively) of horizontal electric field at stratospheric altitudes derived from $y$ probes obtained from every payload spin duration between 04:12 and 05:30 IST. In the bottom panel, the phases derived from $x$ probes are also shown for comparison. Data from the $x$ probes prior to 04:30 IST were noisy and phase estimates for those times are not shown.

\section{Discussion}

As mentioned earlier, the intended goal of the first BEENS experiment was to demonstrate that weak horizontal electric fields at stratospheric altitudes could be captured. A unique configuration of the geomagnetic field at the Equator permits a variety of electrodynamical phenomena to occur in the $\mathrm{E}$ and $\mathrm{F}$ regions of the ionosphere. The equatorial ionosphere is characterized by major features like the plasma fountain responsible for the large-scale redistribution of the ionization during daytime, generation of a spectrum of ionospheric irregularities in the dusk sector and the development of intense current system during daytime called the equatorial electrojet (Kelley, 2009, and references therein), all of which are of significant interest to the scientific community and whose understanding contributes to the subject matter of space weather. As electric fields are the major drivers for these phenomena, the importance of monitoring them cannot be overemphasized.

The payload for the BEENS experiment was designed with the presumption that electric fields at low latitudes were expected to be small (around a few $\mathrm{mV} \mathrm{m}^{-1}$ ). The design envisaged the use of $3 \mathrm{~m}$ long booms for the horizontal probes with a probe-to-probe separation of $\sim 7 \mathrm{~m}$. For the outer $1 \mathrm{~m}$ portion of each of the horizontal booms, insulating fiberglass rods were to be used. However, for the inclined boom used to support the probe intended for vertical electric field measurements, no such insulating arm was proposed. Along with the probe configuration that permitted use of five probes instead of six, implementation of this design had placed constraints on the usefulness of the electric field estimates obtained from $x$ and $z$ probes. There were issues with the motor used for

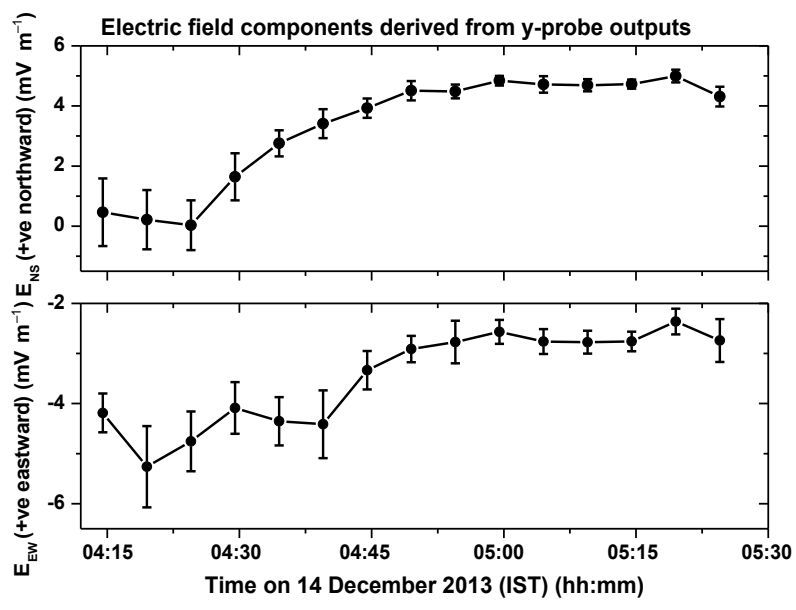

Figure 15. The $5 \mathrm{~min}$ averages of electric field components derived from the horizontal probe outputs, the northward component or $\boldsymbol{E}_{\mathrm{NS}}$ plotted in the top panel and the eastward component or $\boldsymbol{E}_{\mathrm{EW}}$ plotted in the bottom panel. The error bars represent the standard deviation for the electric field estimates made for the individual 5 min blocks. It should be noted that the east-west and north-south directions are referenced in the geomagnetic frame.

spinning the payload that restricted useful electric field measurements to less than $2 \mathrm{~h}$ out of a possible $4.5 \mathrm{~h}$ at the float altitude. Also, when the power supply to the motor was cut off for a brief interval, we detected a perceptible shift in the DC offset and a reduction in the amplitude of the difference output. The motor, when powered, thus possibly induced an additional response in each of the electrometer channels. The authors intend to address these deficiencies and shortcomings in the next flight. Further, any noise environment around the motor and its power supply will have to be examined carefully before launch, and remedial measures are to be taken in order to minimize any electromagnetic noise emanating from them.

We now turn our attention towards the electric field measurements reported in this work. The modulation of the $y$ probe difference output by the payload rotation is quite clear during the $90 \mathrm{~min}$ duration considered for analysis when the rotation rate was uniform (see Figs. 9 and 13). We believe that unlike $x$ probes the electric field estimates obtained from the $y$ probes were not influenced by the deployable boom configuration chosen for the $z$ probes. It may be noted that the latter might contribute to a distortion of the field around the $x$ probe $\left(\mathrm{P}_{1}\right)$ underneath the $z$ probe $\left(\mathrm{P}_{5}\right)$ but not around either of the $y$ probes $\left(\mathrm{P}_{3}\right.$ and $\left.\mathrm{P}_{4}\right)$ (see Fig. 1a for the probe geometry). Probes $\mathrm{P}_{3}$ and $\mathrm{P}_{4}$ were well away and were electrically isolated from rest of the probes and their supporting structures.

During the flight, the power to the DC torque motor was cut off for a brief time interval $(\sim 3 \mathrm{~min})$ as an exercise to see whether the gondola would slow down in its spinning motion and whether there would be an impact on the electric 
field measurements. During the post-launch analysis, it was noticed that the difference output in all three channels did change. However, the electric field signal recorded in the $x$ and $y$ channels retained its character except for a reduction in the amplitude. We used the electric field values estimated for times when the power supply was turned off (the payload continued to spin, though) in order to scale the electric field during rest of the times. The recalculated electric field was in the range $4-6 \mathrm{mV} \mathrm{m}^{-1}$. A noticeable feature of the observations was the similar behavior of the phases estimated from the $x$ and $y$ probe outputs (refer to Fig. 14), which gave us confidence in the measurements reported herein. Reaffirming that electric field measurements of $\sim 5 \mathrm{mV} \mathrm{m}^{-1}$ reported in this work are real, we now proceed to examine the source of the electric field measured by the pair of $y$ probes.

Observing horizontal fields of ionospheric wind dynamo origin from a balloon platform at low to midlatitudes has always been a challenge, because they are smaller (around a few $\mathrm{mV} \mathrm{m}^{-1}$ ) and are difficult to measure. Gonzalez et al. (1982) reported large horizontal electric fields (up to $10 \mathrm{mV} \mathrm{m}^{-1}$ ) in the Brazilian Magnetic Anomaly region. To explain this feature, they invoked the idea that energetic particle precipitation, associated with enhanced magnetic activity, induced irregularities in middle-atmospheric conductivity that in turn produced distortion in the fair-weather vertical electric field and a corresponding change in the horizontal electric field. Orographic features have been known to produce similar irregularities in the conductivity profile that are capable of explaining large horizontal fields observed on some occasions (Ogawa et al., 1975, for example). Such large induced fields would clearly overshadow the weak ionospheric fields expected at balloon altitudes.

As noted earlier, there are no marked orographic features in the vicinity of the balloon track, and therefore we do not expect any enhanced electric field of orographic nature to appear in the experimental data. Further, the results presented in this work pertain to the float altitude of $\sim 35 \mathrm{~km}$, well above the altitude region over which orographic effects are expected to be pronounced. Also, fair-weather conditions prevailed throughout the experiment duration on the ground and just above the ground. The Kalpana satellite imagery does not reveal any high-altitude convective cloud system over the southern Indian peninsular region at the time of the balloon observations reported herein, nor do the infrared images obtained from the EUMETSAT fleet of satellites (refer to Fig. 6). No orographic or local thunderstorm activity effects seemed to have contributed to the observed electric field signatures. Further, there was low to moderate geomagnetic activity around these times. The 3-hourly $\mathrm{Kp}$ index registered a value of $1^{-}$and the Dst index was in the range of 20-25 nT (positive values) during the corresponding time interval. The ground geomagnetic records from low latitudes revealed weak pulsations $(2-4 \mathrm{nT})$ in the period range of 10-20 min. However, as the geomagnetic activity picked up several hours later, it was unlikely that the observed electric fields were related to the geomagnetic activity.

Moving on to atmospheric sources, Holzworth (1989) first reported large horizontal electric fields at balloon altitudes from campaigns conducted during the austral summers of 1983 and 1984 involving long-duration superpressure balloons. The detected fields were in the range of $10-20 \mathrm{mV} \mathrm{m}^{-1}$ during nighttime and $30-70 \mathrm{mV} \mathrm{m}^{-1}$ during daytime at altitudes of $\sim 27 \mathrm{~km}$. Holzworth (1989) referred to such fields as HITS, implying electric fields that were horizontal, inertial, turbulent and stratospheric. A striking feature of those fields was that the horizontal vector rotated counterclockwise at a period that matched the inertial period of a class of atmospheric gravity waves present at those latitudes. The existence of such fields was confirmed in a later work of $\mathrm{Hu}$ and Holzworth (1997) that was carried out from an extended data set covering a wide range of latitudes from the South Pole to $28^{\circ} \mathrm{S}$.

As quiet-time ionospheric electric fields at low latitudes are expected to be in the range of $1-3 \mathrm{mV} \mathrm{m}^{-1}$, we suggest atmospheric gravity waves to be a potential source for the generation of electric fields of magnitudes in the range of 4-6 $\mathrm{mV} \mathrm{m}^{-1}$ reported in this work. Indeed, horizontal winds driven by atmospheric gravity waves can transport charges, and in this process polarization electric fields can be generated in order for the current to vanish. Following the arguments presented in Holzworth (1989) and D'Angelo (1990), the scenario can be elaborated as follows.

Because the vertical electric field, which is part of the GEC, has a gradient with altitude, there arises a net charge density. Use of Gauss's law and taking into account the exponential decrease of the potential gradient with altitude with a scale height of $6 \mathrm{~km}$, we arrive at a net charge density of $\sim 4 \times 10^{-16} \mathrm{C} \mathrm{m}^{-3}$ at an altitude of $35 \mathrm{~km}$. Horizontal winds, associated with atmospheric gravity waves, for example, can transport this charge by advection and thereby drive a current. As the charge density does not vary with time, based on the current continuity principle, we expect the divergence of current in the horizontal direction or, in particular, the current itself to vanish. The latter requirement results in the appearance of polarization electric field that would ensure current balance (refer to Eqs. 1 and 2 in D'Angelo, 1990). The relevant expression for the electric field is $E=\rho U / \sigma$, where $\rho$ is the charge density, $U$ is the wind and $\sigma$ is the conductivity. For a conductivity of $8 \times 10^{-11} \mathrm{~S} \mathrm{~m}^{-1}$ at $35 \mathrm{~km}$ and for a wind speed of $50 \mathrm{~m} \mathrm{~s}^{-1}$, the electric field generated in this way would be $\sim 0.25 \mathrm{mV} \mathrm{m}^{-1}$, which is indeed small. However, it is possible that the sources are located at lower altitudes and the fields generated therein map upward to the balloon float altitude with an attenuation factor that would depend on the horizontal scale size of the generated field. For scale sizes greater than a few hundred kilometers, the attenuation factor simplifies to $e^{-\Delta z / H}$, where $\Delta z$ is the distance from the balloon altitude to where the field is generated (D'Angelo, 1990). Considering the source region to be lo- 
cated at $\sim 10 \mathrm{~km}$ and for a wind speed of $10 \mathrm{~m} \mathrm{~s}^{-1}$, the field generated at the source location would be $\sim 1 \mathrm{~V} \mathrm{~m}^{-1}$. This field, when mapped to $35 \mathrm{~km}$ with an attenuation factor of 0.015 , would take up a value of $\sim 15 \mathrm{mV} \mathrm{m}^{-1}$. Thus, even with their modest phase speeds of $10 \mathrm{~m} \mathrm{~s}^{-1}$, gravity waves of horizontal wavelengths of few hundreds of kilometers and time periods in the range of $1-3 \mathrm{~h}$ can generate electric fields of sufficient magnitude to be observable at the balloon float altitude of $\sim 35 \mathrm{~km}$. However, it remains to be seen whether such fields are a persistent feature of the stratosphere at low latitudes.

\section{Conclusion}

The BEENS experiment was carried out from the National Balloon Facility at Hyderabad just prior to the early hours of 14 December 2013. The primary goals of the experiment were to observe stratospheric electric fields at these low latitudes and understand their relationship to the known electric field drivers. The measurements themselves had to be carefully analyzed, as there were shortcomings like the kind of deployable boom used for vertical electric field measurements and the probe geometry adopted, which restricted useful electric field observations to those by one pair of horizontal probes out of two. A malfunctioning of the orientor further restricted the observation duration to $90 \mathrm{~min}$ at the float altitude of $35 \mathrm{~km}$. With the apprehension that the orientor, when powered, possibly introduced a noise field, we had to use a small part of the data set corresponding to times when the power supply to the orientor was switched off through a ground command. In spite of these shortcomings, the presence of a moderate $\sim 5 \mathrm{mV} \mathrm{m}^{-1}$ horizontal electric field at the float altitudes of $\sim 35 \mathrm{~km}$ during the flight could be detected. The experiment had presented us several challenges to be met, and it is hoped that future long-duration flights from these low latitudes will be more scientifically rewarding and offer us enough scope to investigate the role played by atmospheric sources in the generation of electric fields of magnitudes such as reported in this work.

\section{Data availability}

The electric field data used in this work were acquired by the Indian Institute of Geomagnetism (IIG) from a specially designed balloon experiment conducted at the National Balloon Facility (NBF), Tata Institute of Fundamental Research (TIFR), Hyderabad. IIG has a data sharing policy in place, and any request for data can be addressed in line with this policy. The thermal infrared image shown in Fig. 7 is from the archives of the NEODAAS Dundee Satellite Receiving Station, which used the EUMETCast distribution service provided by EUMETSAT.
Competing interests. The authors declare that they have no conflict of interest.

Acknowledgements. The authors gratefully acknowledge TIFR for providing the launch platform and necessary facilities at its Balloon Facility at Hyderabad for conducting this experiment. All balloon operations were carried out by the staff of NBF. The authors are thankful to Robert Holzworth for his useful comments on an earlier design of the payload. The BEENS experiment was carried out with the financial support extended by the Department of Space, Government of India. S. Manu thanks the director of the Indian Institute of Geomagnetism, Navi Mumbai, for a research scholarship, during his association with the Indian Institute of Geomagnetism.

The topical editor, K. Shiokawa, thanks two anonymous referees for help in evaluating this paper.

\section{References}

Bloomfield, P.: Fourier Analysis of Time Series: An Introduction, John Wiley \& Sons, Inc., 2000.

D'Angelo, N.: Comment on "A new source of horizontal electric fields in the mid-latitude stratosphere" by R. H. Holzworth, J. Geophys. Res., 95, 11913-11914, 1990.

Franz, R. C., Nemzek, R. J., and Winckler, J. R.: Television image of a large upward electrical discharge above a thunderstorm system, Science, 264, 48-51, 1990.

Garg, S. C., John, T., Zalpuri, K. S., Subrahmanyam, P., and Somayajulu, V. V.: Measurement of stratospheric electrical conductivity using balloon-borne Langmuir probe, Indian J. Radio Space, 18, 285-289, 1989.

Gonzalez, W. D., Pereira, A. E. C., Golnzalez, A. L. C., Martin, I. M., Dutra, S. L. G., Pinto Jr., O., Wygant, J., and Mozer, F. S.: Large horizontal electric fields measured at balloon heights of the Brazilian magnetic anomaly and association to local energetic particle precipitation, Geophys. Res. Lett., 9, 567-570, 1982.

Gupta, S. P. and Narayan, A.: Balloon-borne measurements of ion conductivity over low latitude stratosphere, Planet. Space Sci., 35, 439-443, 1987.

Hays, P. B. and Roble, R. G.: A quasi-static model of global atmospheric electricity, 1. The lower atmosphere, J. Geophys. Res., 84, 3291-3305, 1979a.

Hays, P. B. and Roble, R. G.: A quasi-static model of global atmospheric electricity, 2. Electrical coupling between the upper and lower atmosphere, J. Geophys. Res., 84, 7247-7256, 1979b.

Holzworth, R. H.: A new source of horizontal electric fields in the mid-latitude stratosphere, J. Geophs. Res., 94, 12795-12802, 1989.

Holzworth, R. H.: Quasistatic electromagnetic phenomena in the atmosphere and ionosphere, in Handbook of Atmospheric Electrodynamics, Vol. 1, CRC Press, Inc., 1995.

Holzworth, R. H. and Bering III, E. A.: Ionospheric electric fields from stratospheric balloon-borne probes, in: Measurement Techniques in Space Plasmas: Fields, Geophys. Monogr. Ser., Vol. 103, edited by: Pfaff, R. F., Borovsky, J. E., and Young, D. T., 79-84, AGU, Washington, D. C., 1998. 
Hu, H. and Holzworth, R. H.: An inertial wave-driven stratospheric horizontal electric field: New evidence, J. Geophys. Res., 102, 19717-19727, 1997.

Jawahar, K., Manu, S., and Gurubaran, S.: A differential electrometer for vector electric field measurements on a balloon platform, Current Sci., 111, 624-626, 2016.

Kelley, M. C.: In The Earth's Ionosphere: Plasma Physics and Electrodynamics, International Geophysics Series, Vol. 96, Academic, 576 pp., 2009.

Mozer, F. S. and Serlin, S., Magnetospheric electric field measurements with balloons, J. Geophys. Res., 74, 4739-4754, 1969.

Ogawa, T., Tanaka, Y., Huzita, A., and Yasuhara, M., Horizontal electric fields in the middle latitude, Planet. Space Sci., 23, 825830, 1975.

Park, C. G.: Downward mapping of high-latitude ionospheric electric fields to the ground, J. Geophys. Res., 81, 168-174, 1976.

Rycroft, M. J. and Odzimek, A.: Effects of lightning and sprites on the ionospheric potential, and threshold effects on sprite initiation, obtained using an analog model of the global atmospheric electric circuit, J. Geophys. Res., 115, A00E37, doi:10.1029/2009JA014758, 2010.
Rycroft, M. J., Nicoll, K. A., Aplin, K. L., and Harrison, R. G.: Recent advances in global electric circuit coupling between the space environment and the troposphere, J. Atmos. Sol.-Terr. Phy., 90-91, 198-211, 2012.

Sampath, S., Murali Das, S., and Sasi Kumar, V.: Electrical conductivities, ion densities and mobilities in the middle atmosphere over India - Balloon measurements, J. Atmos. Terr. Phys., 51, 533-540, 1989.

Sentman, D. D., Wescott, E. M., Osborne, D. L., Hampton, D. L., and Heavner, M. J.: Preliminary results from the Sprites94 Aircraft Campaign: 1. Red sprites, Geophys. Res. Lett., 22, 12051208, 1995.

Udare, R. S., Rajaram, R., Ogawa, T., and Yashuhara, M.: Height profile of vertical electric field and conductivity over Hyderabad, Indian J. Radio Space, 20, 307-309, 1991.

Volland, H.: Mapping of the electric field of the $S_{q}$ current into the lower atmosphere, J. Geophys. Res., 77, 1961-1965, 1972. 